\title{
ON THE PICARD NUMBERS OF ABELIAN VARIETIES
}

\author{
KLAUS HULEK AND ROBERTO LAFACE
}

\begin{abstract}
In this paper we study the possible Picard numbers $\rho$ of an abelian variety $A$ of dimension $g$. It is well known that this satisfies the inequality $1 \leq \rho \leq g^{2}$. We prove that the set $R_{g}$ of realizable Picard numbers of abelian varieties of dimension $g$ is not complete for every $g \geq 3$, namely that $R_{g} \subsetneq\left[1, g^{2}\right] \cap \mathbb{N}$. Moreover, we study the structure of $R_{g}$ as $g \rightarrow+\infty$, and from that we deduce a structure theorem for abelian varieties of large Picard number. In contrast to the non-completeness of any of the sets $R_{g}$ for $g \geq 3$, we also show that the Picard numbers of abelian varieties are asymptotically complete, i.e. $\lim _{g \rightarrow+\infty} \# R_{g} / g^{2}=1$. As a byproduct, we deduce a structure theorem for abelian varieties of large Picard number. Finally we show that all realizable Picard numbers in $R_{g}$ can be obtained by an abelian variety defined over a number field.
\end{abstract}

\section{INTRODUCTION}

For an algebraic variety $X$ over the field of complex numbers the Lefschetz $(1,1)$-theorem says that the Néron-Severi group

$$
\mathrm{NS}(X)=\mathrm{H}^{2}(X, \mathbb{Z}) \cap \mathrm{H}^{1,1}(X) .
$$

Consequently, the rank $\rho(X)$ of the Néron-Severi group, the so-called Picard number, satisfies the inequality $1 \leq \rho(X) \leq h^{1,1}(X)$. Computing the Picard number is in general a difficult question, as already the case of projective surfaces shows. For example, the Picard number of a quintic surface $S$ in $\mathbb{P}^{3}$ satisfies the inequality $\rho(S) \leq 45$. It is known that all numbers between 1 and 45 can be obtained if one allows the surface to have $A D E$-singularities, but it remains an open problem for smooth surfaces, where the maximum known is 41 [15], [16].

In this article we will concentrate on the Picard numbers of abelian varieties. To put this into perspective it is worthwhile to recall the situation for surfaces. For abelian surfaces all possible Picard numbers between 1 (or 0 if one includes the non-algebraic case) and 4 occur. Indeed, a very general abelian surface has $\rho=1$, whereas Picard numbers from 2 to 4 can be realized by taking a product $E_{1} \times E_{2}$ of two elliptic curves. If the two elliptic curves are not isogenous, then $\rho=2$, if they are isogenous but they do not have complex multiplication, then $\rho=3$, while if they also have complex multiplication $\rho=4$. For the other surfaces with trivial canonical bundle the situation is similar: for K3 surfaces all possibilities between 1 (respectively 0) and 20 can occur, as can be seen by the Torelli theorem for K3 surfaces. Enriques surfaces and bi-elliptic surfaces have no holomorphic 2-forms, and thus their Picard number is 10 and 2 respectively.

For higher-dimensional varieties with numerically trivial canonical bundle the situation is as follows. By the Beauville-Bogomolov decomposition theorem [4], every Kähler manifold with trivial first Chern class admits a finite cover which is a product of tori, Calabi-Yau varieties and irreducible holomorphic symplectic manifolds (IHSM), also know as hyperkähler manifolds. For higher dimensional Calabi-Yau varieties $Y$ we always have $\rho(Y)=b_{2}(Y)$ as $h^{2,0}(Y)=h^{0,2}(Y)=0$. For irreducible holomorphic symplectic manifolds $X$ one can use 
Huybrechts' surjectivity of the period map [11] to conclude, as in the case of K3 surfaces, that all values $0 \leq \rho(X) \leq b_{2}(X)-2$ can be obtained. This leaves us with the case of abelian varieties which is the topic of this note. Surprisingly little seems to be known about the possible Picard numbers of abelian varieties. Our aim is to make a first start to remedy this situation, using mostly elementary methods.

Let $A$ be a complex torus of dimension $g$. Its cohomology is the exterior algebra over $\mathrm{H}^{1}(A, \mathbb{Z}) \cong \mathbb{Z}^{2 g}$. In particular, this implies that the $k^{\text {th }}$ Betti numbers are $b_{k}(A)=\left(\begin{array}{c}2 g \\ k\end{array}\right)$. As $\mathrm{H}^{p, 0}(A) \cong \mathrm{H}^{0}\left(A, \Omega_{A}^{p}\right)$, we get $h^{p, 0}(A)=\left(\begin{array}{l}g \\ p\end{array}\right)$, and thus $h^{1,1}(A)=g^{2}$. We shall from now on exclude the case of non-algebraic tori and concentrate on abelian varieties. By the above we know that

$$
1 \leq \rho \leq g^{2}
$$

As we have already seen, any number $1 \leq \rho(A) \leq 4$ can be achieved for abelian surfaces. However, the situation changes significantly in higher dimension.

Given an arbitrary abelian variety $A$, we can invoke Poincaré Complete Reducibility Theorem [7, Thm. 5.3.7] to pass to a better representative in its isogeny class, namely

$$
A \longrightarrow A_{1}^{n_{1}} \times \cdots \times A_{r}^{n_{r}}
$$

where $A_{i}$ is a simple abelian variety $(i=1, \ldots, r)$, and $A_{i}$ is not isogenous to $A_{j}$ if $i \neq j$. Moreover, the abelian varieties $A_{i}$ and the integers $n_{i}$ are uniquely determined up to isogeny and permutations. A result of Murty [12, Lemma 3.3] describes the Picard number of a self-product $B^{k}$ of a simple abelian variety in terms of $k$ and the dimension of $B$. In light of this, we prove in Proposition 2.2 a splitting result concerning the Picard group of varieties of the form $A \times B$ with $\operatorname{Hom}(A, B)=0$, which allows us to compute the Picard number of such products. This, together with Murty's result in [12], provides us with a theoretical algorithm for computing the Picard number of a given abelian variety.

It is then a combinatorial question as to determine the set $R_{g}$ of possible Picard numbers of abelian varieties for a given genus $g$. Very little seems to be known about this. The purpose of this paper is to take a first step in the analysis of $R_{g}$. As a first result we show that there are gap series for the possible Picard numbers of abelian varieties, and therefore that the sets $R_{g}$ are not complete for every $g \geq 3$. In fact, it is not hard to show that $R_{3}=\{1, \ldots, 6,9\}$ : indeed, a very general abelian threefold has Picard number $\rho=1$; a product $S \times E, S$ being a very general abelian surface and $E$ being an elliptic curve, has Picard number $\rho=2$; all other Picard numbers can be obtained by using suitable products of elliptic curves. This phenomenon had previously been notice by Shioda in [21, Appendix].

As the dimension $g$ grows larger, clear gaps in the set of possible Picard numbers start to appear. Moreover, more and more gaps occur as $g \rightarrow \infty$. The following result shows the existence of two precise gaps and characterizes the three largest Picard numbers for an abelian variety.

\section{Theorem 1.1.}

(1) Fix $g \geq 4$. There does not exist any abelian variety of dimension $g$ with Picard number $\rho$ in the following range:

$$
(g-1)^{2}+\underset{2}{1}<\rho<g^{2} .
$$


(2) Fix $g \geq 7$. There does not exist any abelian variety of dimension $g$ with Picard number $\rho$ in the following range:

$$
(g-2)^{2}+4<\rho<(g-1)^{2}+1 .
$$

We would like to remark that the conditions on the dimension $g$ given in Part 1 and 2 of Theorem 1.1 are necessary. In fact, as for Part 1, for $g=2$ all Picard numbers occur, and for $g=3$ there exists an abelian threefold of Picard number $\rho=6$ (namely, the product of three isogenous elliptic curves without complex multiplication). Similar considerations can be made for Part 2 of Theorem 1.1 and $g \leq 6$. After some preliminary work in Section 2, we shall prove this theorem in Section 3. As an application of our analysis we derive in Section 4 a structure theorem for abelian varieties with large Picard number, namely Theorem 4.2 .

The above results are a first indication of a much more general phenomenon which we study more systematically in Section 7, where we consider the behaviour of the set $R_{g}$ asymptotically, namely as $g$ grows. In particular, we define the asymptotic density of Picard numbers to be the quantity

$$
\delta:=\lim _{g \rightarrow+\infty} \frac{\# R_{g}}{g^{2}} .
$$

Contrary to the non-completeness of any of the sets $R_{g}$, we prove asymptotic completeness in Theorem 7.1, namely

Theorem 1.2. The Picard numbers of abelian varieties are asymptotically complete:

$$
\delta=\lim _{g \rightarrow+\infty} \frac{\# R_{g}}{g^{2}}=1
$$

By using similar techniques, we are also able to describe the distribution of the Picard numbers within $\left[1, g^{2}\right] \cap \mathbb{N}$ (Theorem 7.4 ). As a consequence, we obtain a structure theorem for abelian varieties of large Picard number in Corollary 7.6. We also provide a practical algorithm which allows to compute the sets $R_{g}$ inductively.

Finally we show that all realizable Picard numbers $\rho \in R_{g}$ can be obtained by an abelian variety defined over a number field.

Acknowledgement. We would like to thank Bert van Geemen for his genuine interest in this question, and Matthias Schütt for discussions around this topic and beyond. RL would like to particularly thank François Charles for the many fruitful discussions and for his invitation to the IHÉS, whose excellent working conditions are here gratefully acknowledged. We would like to thank Davide Lombardo for suggesting the proof of Lemma 7.2. We gratefully acknowledge a very fruitful exchange of ideas with Ben Moonen who also supplied the proof of Theorem 8.1. Finally, we are grateful to the anonymous referee for his or her comments, which have helped improving the manuscript. This research was partially funded by the DFG funded GRK 1463 "Analysis, Geometry and String Theory".

\section{Preliminary WORK}

In this section we will develop the basic tools of our analysis. Some of these results are of independent interest in their own right. 
2.1. Additivity of the Picard number for non-isogeneous products. As the Picard number of an abelian variety is invariant under isogenies [6, Ch. 1, Prop. 3.2], we can pick a convenient representative in its isogeny class. Such a choice is indicated by the following result [7, Thm 5.3.7]:

Theorem 2.1 (Poincaré's Complete Reducibility Theorem). Given an abelian variety A, there exists an isogeny

$$
A \longrightarrow A_{1}^{n_{1}} \times \cdots \times A_{r}^{n_{r}}
$$

where $A_{i}$ is a simple abelian variety $(i=1, \ldots, r)$, and $A_{i}$ is not isogenous to $A_{j}$ if $i \neq j$. Moreover, the abelian varieties $A_{i}$ and the integers $n_{i}$ are uniquely determined up to isogeny and permutations.

Let us now consider a product of simple abelian varieties as in Theorem 2.1. The fact that $A_{i}$ is not isogenous to $A_{j}$ for $i \neq j$ yields the following splitting of the Picard group:

Proposition 2.2. Let $A_{1}, \ldots, A_{r}$ be simple abelian varieties, such that $A_{i}$ is not isogenous to $A_{j}$ for $i \neq j$. Then the (exterior) pullback of line bundles yields an isomorphism

$$
\prod_{i=1}^{r} \operatorname{Pic}\left(A_{i}^{n_{i}}\right) \cong \operatorname{Pic}\left(\prod_{i=1}^{r} A_{i}^{n_{i}}\right) .
$$

Clearly, exterior pull-back of line bundles always yields an injective map, but surjectivity is a special feature. In fact, if $E$ is an elliptic curve, the abelian surface $E \times E$ has Picard number $\rho \in\{3,4\}$, depending on the presence of CM. Therefore, the exterior pull-back map

$$
\operatorname{Pic}(E) \times \operatorname{Pic}(E) \longrightarrow \operatorname{Pic}(E \times E)
$$

cannot be surjective, as otherwise we would get a surjective map of the corresponding NéronSeveri groups, hence yielding a contradiction, since $\mathrm{NS}(E) \cong \mathbb{Z}$.

Proof. Exterior pullback of line bundles

$$
\psi\left(L_{1}, \ldots, L_{r}\right)=L_{1} \otimes \cdots \otimes L_{r}
$$

defines the following commutative diagram

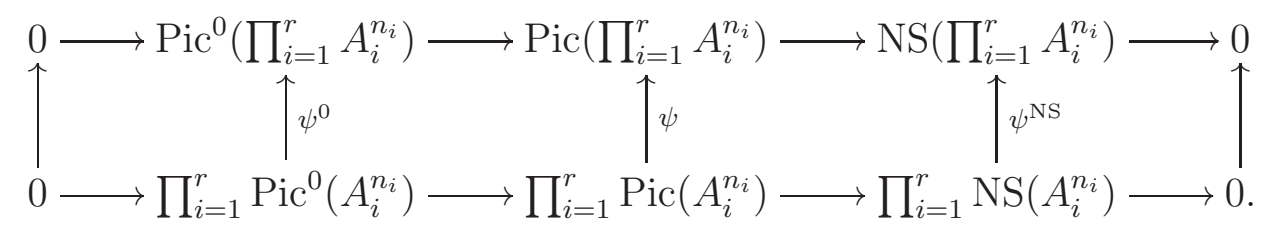

We will show that $\psi^{0}$ and $\psi^{\text {NS }}$ are isomorphisms, thus proving the proposition. Clearly $\psi^{0}$ is injective, and since $\psi^{0}$ is a homomorphism of abelian varieties of the same dimension it must be an isomorphism. To prove that $\psi^{\mathrm{NS}}$ is an isomorphism we recall from [7, Ch. 2] that a polarization on an abelian variety $A$ is given by a finite isogeny $f: A \rightarrow A^{\vee}$ whose analytic representation is hermitian. By assumption the abelian varieties $A_{i}$ and $A_{j}$ are not isogeneous for $i \neq j$. Hence $\operatorname{Hom}\left(A_{i}, A_{j}\right)=\operatorname{Hom}\left(A_{i}, A_{j}^{\vee}\right)=0$ and every isogeny

$$
f: \prod_{i=1}^{r} A_{i}^{n_{i}} \longrightarrow\left(\prod_{i=1}^{r} A_{i}^{n_{i}}\right)^{\vee}
$$


is of the form $f=\left(f_{1}, \ldots, f_{r}\right)$ where $f_{i}: A_{i}^{n_{i}} \longrightarrow\left(A_{i}^{n_{i}}\right)^{\vee}$ is an isogeny. Since a direct sum of endomorphisms is hermitian if and only if all its summands are, the claim follows, as every class in the Néron-Severi group can be written as the difference of two ample classes (i.e. two polarizations).

As a consequence, we get that the Picard number is additive (but not strongly additive) for product varieties coming from the Poincaré's Complete Reducibility Theorem.

Corollary 2.3. Let $A_{1}, \ldots, A_{r}$ be simple abelian varieties, such that $A_{i}$ is not isogenous to $A_{j}$ for $i \neq j$. Then,

$$
\rho\left(\prod_{i=1}^{r} A_{i}^{n_{i}}\right)=\sum_{i=1}^{r} \rho\left(A_{i}^{n_{i}}\right) .
$$

2.2. Picard numbers of self-products. Due to additivity, we are left to see how to compute the Picard number in the case of a self-product of a simple abelian variety. The endomorphism ring $\operatorname{End}(A)$ of an abelian variety $A$ is a finitely generated free abelian group and hence $F:=\operatorname{End}(A) \otimes \mathbb{Q} \equiv \operatorname{End}_{\mathbb{Q}}(A)$ is a finite dimensional $\mathbb{Q}$-algebra. Any polarization $L$ on $A$ defines an involution on $F$, the so-called Rosati involution. If $A$ is a simple abelian variety, then $F$ is a finite-dimensional skew field admitting a positive anti-involution. Such pairs were classified by Albert [1], 3], see also [7, Proposition 5.5.7] for a summary.

Let $K$ be the centre of $F$. We will say that $F$ is of the first kind if the Rosati involution acts trivially on $K$, and of the second kind otherwise. Let us denote by $K_{0}$ the maximal real subfield of $K$, and let us consider the following invariants of $F$ :

$$
[F: K]=d^{2}, \quad[K: \mathbb{Q}]=e, \quad\left[K_{0}: \mathbb{Q}\right]=e_{0} .
$$

Notice that, as $K$ is the center of $F,[F: K]$ is always a square.

As a useful example, we can consider quaternion algebras over a field $K$. Let us recall the reader that any quaternion algebra $F / K$ is either a division ring or it is isomorphic to $M_{2}(K)$. In light of this, we can define the ramification locus of $F$ as

$$
\operatorname{Ram}(F)=\left\{\mathfrak{p} \in \operatorname{Spec} \mathcal{O}_{K} \mid K_{\mathfrak{p}} \text { is a division ring }\right\} .
$$

A quaternion algebra is ramified at a finite even number of places. The ramification locus governs the isomorphism classes of quaternion algebras: there is a 1:1 correspondence between isomorphism classes of quaternion algebras and subsets of non-complex places of $K$ of even cardinality.

By [7, Proposition 5.5.7] the classification divides into four types, where the first three are of the first kind:

(1) Type $I: F$ is a totally real number field, so that $d=1$ and $e=e_{0}$.

(2) Type $I I: F$ is a totally indefinite quaternion algebra over a totally real number field $K$, i.e.

$$
\emptyset=\operatorname{Ram}(F) \cap\{\operatorname{archimedean} \text { places of } K\} \quad \text { and } \quad \operatorname{Ram}(F) \neq \emptyset .
$$

In particular, we have that $d=2$ and $e=e_{0}$.

(3) Type III: $F$ is a totally definite quaternion algebra over $K$, i.e.

$$
\emptyset \neq \operatorname{Ram}(F) \supseteq\{\operatorname{archimedean} \text { places of } K\}
$$

holds. Again, we have $d=2$ and $e=e_{0}$. 
(4) Type $I V: F$ is of the second kind, and it center $Z(F)=K$ is a CM field with maximal real subfield $K_{0}$.

The following result, due to Murty, gives a complete description of the Picard number of a self-product of a simple abelian variety.

Proposition 2.4 (Lemma 3.3 of [12]). Let $A$ be a simple abelian variety. Set $e:=[K: \mathbb{Q}]$, $d^{2}:=[F: K]$. Then, for $k \geq 1$, one has

$$
\rho\left(A^{k}\right)= \begin{cases}\frac{1}{2} e k(k+1) & \text { Type } I \\ e k(2 k+1) & \text { Type II } \\ e k(2 k-1) & \text { Type III } \\ \frac{1}{2} e d^{2} k^{2} & \text { Type IV. }\end{cases}
$$

In fact, Murty's result is in terms of the maximal commutative subfield $E$ of $F$, which has degree $[E: K]$ over $K$. However, the proof of [12, Lemma 2.2] implies that $[E: K]^{2}=[F: K]$. Proposition 2.4 enables us to compute the following bound for the Picard number of a selfproduct of a simple abelian variety:

Corollary 2.5. Let $A$ be a simple abelian variety of dimension $n$, and let $k \geq 1$. Then $\rho\left(A^{k}\right) \leq \frac{1}{2} n k(2 k+1)$.

Proof. Proposition 2.4 applied with $k=1$ allows us to compute the Picard number of $A$ :

$$
\rho=\rho(A)= \begin{cases}e & \text { Type } I \\ 3 e & \text { Type II } \\ e & \text { Type III } \\ \frac{1}{2} e d^{2} & \text { Type IV. }\end{cases}
$$

Now, plugging this back in Proposition 2.4 gives the following reformulation in terms of the Picard number of $A$ :

$$
\rho\left(A^{k}\right)= \begin{cases}\frac{1}{2} \rho k(k+1) & \text { Type } I \\ \frac{1}{3} \rho k(2 k+1) & \text { Type } I I \\ \rho k(2 k-1) & \text { Type III } \\ \rho k^{2} & \text { Type } I V .\end{cases}
$$

The divisibility conditions for $\rho$ given by [7, Prop. 5.5.7] imply that

$$
\rho \leq \begin{cases}n & \text { Type } I \\ \frac{3}{2} n & \text { Type II } \\ \frac{1}{2} n & \text { Type III } \\ n & \text { Type IV }\end{cases}
$$

and, therefore, we see that

$$
\rho\left(A^{k}\right) \leq \begin{cases}\frac{1}{2} n k(k+1) & \text { Type } I \\ \frac{1}{2} n k(2 k+1) & \text { Type II } \\ \frac{1}{2} n k(2 k-1) & \text { Type III } \\ n k^{2} & \text { Type IV }\end{cases}
$$

from which the result follows. 
In the case of a self-product of an elliptic curve this gives the well known

Corollary 2.6. If $E$ is an elliptic curve, then

$$
\rho\left(E^{k}\right)= \begin{cases}\frac{1}{2} k(k+1) & \text { E has no CM } \\ k^{2} & \text { E has CM. }\end{cases}
$$

We will use these results, in particularly the case of a self-product of elliptic curves, frequently in the proof of Theorem 1.1. Notice that Corollary 2.5 provides us with a bound on the Picard number of $A^{k}$ which is independent of the type of the endomorphism ring of A.

\section{Restrictions on the Picard number}

3.1. Some bounds on the Picard number. We would like to show that there are better bounds on the Picard number, if one is given a partition of the dimension. More precisely, letting $A$ be an abelian variety, we define $r(A)$ to be the length of a decomposition according to Poincaré Complete Reducibility Theorem. In other words, given an abelian variety $A$, Theorem 2.1 gives an isogeny

$$
A \longrightarrow A_{1}^{n_{1}} \times \cdots \times A_{r}^{n_{r}}
$$

and we set $r(A):=r$. Notice that this quantity is well-defined because the factors $A_{i}$ and the powers $n_{i}$ are determined up to permutations and isogenies. Then, for $r \leq g$, we define $M_{r, g}$ as

$$
M_{r, g}:=\max \{\rho(A) \mid \operatorname{dim} A=g, r(A)=r\} .
$$

In other words, $M_{r, g}$ is the largest Picard number that can be realized by a $g$-dimensional abelian variety that splits into a product of $r$ non-isogenous pieces in its isogeny class.

Proposition 3.1. For integers $r, g \in \mathbb{N}$ such that $r \leq g$, one has $M_{r, g}=[g-(r-1)]^{2}+(r-1)$. This value is attained as the Picard number of $E^{g-r+1} \times E_{1} \times \cdots \times E_{r-1}$, where $E$ is a $C M$ elliptic curve not isogenous to any of the $E_{i}$ 's, and $E_{i}$ and $E_{j}$ are not isogenous for $i \neq j$.

Proof. If $A \sim A_{1} \times \cdots \times A_{r}, \operatorname{Hom}\left(A_{i}, A_{j}\right)=0$ for $i \neq j$, then

$$
\rho(A) \leq k_{1}^{2}+\cdots+k_{r}^{2}
$$

where $k_{i}:=\operatorname{dim} A_{i}(i=1, \ldots, r)$ and $k_{1}+\cdots+k_{r}=g$. Hence we are looking for the maxima of the function

$$
h\left(x_{1}, \ldots, x_{r}\right)=x_{1}^{2}+\cdots+x_{r-1}^{2}+x_{r}^{2}
$$

on the integral points of the simplex

$$
\Omega_{r, g}=\left\{\left(x_{1}, \ldots, x_{r}\right) \mid x_{i} \geq 1, x_{1}+\ldots x_{r}=g\right\} .
$$

These points are precisely the vertices

$$
\{(g-r+1,1, \ldots, 1),(1, g-r+1,1, \ldots, 1), \ldots,(1, \ldots, 1, g-r+1)\} .
$$

By the symmetry of $h$, the maximum is attained at any of these points, with value

$$
h(g-r+1,1, \ldots, 1)=[g-(r-1)]^{2}+(r-1) .
$$

Therefore, we conclude that $\rho(A) \leq[g-(r-1)]^{2}+(r-1)$. By applying Proposition 2.2 and Corollary 2.6 one can see that the abelian variety

$$
E^{g-r+1} \times E_{1} \times \cdots \times E_{r-1}
$$


with $E$ a $\mathrm{CM}$ curve and $E, E_{i}, E_{j}$ for $i \neq j$ not pairwise mutually isogeneous, has Picard number $[g-(r-1)]^{2}+(r-1)$, and we are thus done.

Corollary 3.2. Let $A$ be an abelian variety. Then,

$$
\rho(A)=M_{r(A), g} \Longleftrightarrow A \sim E^{g-(r-1)} \times E_{1} \times \cdots \times E_{r-1},
$$

where $E$ is a $C M$ elliptic curve not isogenous to any of the $E_{i}$ 's, and $E_{i}$ and $E_{j}$ are not isogenous for $i \neq j$.

Remark 3.3. The numbers $M_{r, g}$ give the following (strictly) increasing sequence of positive integers:

$$
g=M_{g, g}<M_{g-1, g}<\cdots<M_{3, g}<M_{2, g}<M_{1, g}=g^{2} .
$$

We will now proceed with the proof of Theorem 1.1, which we divide into two parts.

3.2. Proof of part (1). Let $A$ be an abelian variety of dimension $g \geq 4$ with Picard number $\rho=\rho(A)$. We will divide our analysis of the Picard number $\rho$ into the following cases:

(a) $A$ has length at least two, i.e. $r(A) \geq 2$;

(b) $A$ is a self-product of a lower dimensional abelian variety.

Case (a). Since $r(A) \geq 2$, we have that $A \sim A_{1} \times A_{2}$ with $\operatorname{Hom}\left(A_{1}, A_{2}\right)=0$. Let $n:=\operatorname{dim} A_{1}$, so that $\operatorname{dim} A_{2}=g-n$. Then, $\rho(A) \leq n^{2}+(g-n)^{2}$. Consider the function

$$
f(x):=x^{2}+(g-x)^{2}
$$

on $\Omega=[1, g-1]$. It attains its maximum at $x=1$ and $x=g-1$, with value $f(1)=$ $f(g-1)=(g-1)^{2}+1$. Therefore, $\rho(A) \leq(g-1)^{2}+1$.

Case (b). Let $B$ be an $m$-dimensional simple abelian variety, and suppose $A$ is isogenous to $B^{k}$, for $k:=g / m$. If $m=1$ (i.e. $B$ is an elliptic curve), then again by Corollary 2.6

$$
\rho\left(B^{g}\right)= \begin{cases}\left(\begin{array}{c}
g+1 \\
2
\end{array}\right) & B \text { has no CM } \\
g^{2} & B \text { has CM. }\end{cases}
$$

If $B$ has CM, then $A$ attains the maximal Picard number $g^{2}$; if $B$ does not have CM, then

$$
\rho(A)=\left(\begin{array}{c}
g+1 \\
2
\end{array}\right) \leq 1+(g-1)^{2}
$$

because $g \geq 4$. The case of a self-product of an elliptic curve being dealt with, we can assume $k \leq g / 2$. Then, by Corollary 2.5 we have

$$
\rho\left(B^{k}\right) \leq \frac{1}{2} g(2 k+1) \leq \frac{1}{2} g(g+1)
$$

and the claim follows, since the equality

$$
\frac{1}{2} g(g+1) \leq(g-1)^{2}+1
$$

holds for $g \geq 4$. 
3.3. Proof of part (2). To start with observe that, if $r(A) \geq 3$, then

$$
\rho(A) \leq M_{r(A), g} \leq M_{3, g}<(g-2)^{2}+4 .
$$

Therefore, we can assume $r(A) \leq 2$. Suppose that $A$ is an abelian variety with $r(A)=1$, i.e. $A \sim B^{k}$ with $\operatorname{dim} B=b$ and $b k=g$. If $b=1$, then $B$ is an elliptic curve and we have two cases according to whether $B$ has complex multiplication. If $B$ does have complex multiplication, then $\rho(A)=g^{2}$ (the maximal Picard number), otherwise $\rho(A)=\frac{1}{2} g(g+1)<(g-2)^{2}+4$ (as $g \geq 7$ ). If $b>1$, then $k \leq g / 2$ and thus, by Corollary 2.5.

$$
\rho\left(B^{k}\right) \leq \frac{1}{2} g(2 k+1) \leq \frac{1}{2} g(g+1) \leq(g-2)^{2}+4
$$

again because $g \geq 7$. The last remaining case is $r(A)=2$, which we will divide into three steps.

Step 1. We deal with abelian varieties of the form $E_{1}^{n} \times E_{2}^{g-n}$, where $E_{1}$ and $E_{2}$ are elliptic curves, and $1 \leq n \leq g-n$. If $n=1$, then, by Proposition 2.3

$$
\rho\left(E_{1} \times E_{2}^{g-1}\right)=1+\rho\left(E_{2}^{g-1}\right)
$$

which equals $M_{2, g}$ if $E_{2}$ has complex multiplication, and $1+\frac{1}{2} g(g-1)$ otherwise. In the CM case, we obtain the second largest attainable Picard number, in the non-CM case instead one sees that it is always the case that $1+\frac{1}{2} g(g-1) \leq(g-2)^{2}+4$. Suppose now that $n \geq 2$ : we have that $\rho\left(E_{1}^{n} \times E_{2}^{g-n}\right) \leq n^{2}+(g-n)^{2}$, and we want to bound the right-hand side. The function

$$
f(x):=x^{2}+(g-x)^{2}
$$

attains its maximum on the interval $\Omega=[2, g-2]$ at $x=2$ and $x=g-2$ with value $f(2)=f(g-2)=(g-2)^{2}+4$. This implies that $\rho\left(E_{1}^{n} \times E_{2}^{g-n}\right) \leq(g-2)^{2}+4$.

Step 2. We now consider abelian varieties of the form $E^{k} \times A^{l}$, with $E$ an elliptic curve, $\operatorname{dim} A=a>1, k \geq 1, l \geq 1$ and $g=k+a l$. Notice that, by Proposition 2.3 and Lemma 2.5, one has

Consider the function

$$
\rho\left(E^{k} \times A^{l}\right) \leq k^{2}+\frac{1}{2} a l(2 l+1)=k^{2}+\frac{1}{2}(g-k)(2 l+1) .
$$

$$
f(x, y)=x^{2}+\frac{1}{2}(g-x)(2 y+1),
$$

in the domain $\Omega:=\left\{(x, y) \in \mathbb{R}^{2} \mid x \geq 1, y \geq 1, x+2 y \leq g\right\}$. We will prove that $f$ is bounded from above by $(g-2)^{2}+4$ in $\Omega$.

By looking at the partials

$$
\frac{\partial f}{\partial x}(x, y)=2 x-y-\frac{1}{2}, \quad \frac{\partial f}{\partial y}(x, y)=g-x,
$$

we see that $f$ is increasing on the lines where $x$ is constant. Thus the maximum of $f$ in $\Omega$ will lie on the line $x+2 y=g$. Therefore, we have reduced ourselves to studying the function

$$
g(y):=f(g-2 y, y)=(g-2 y)^{2}+2 y^{2}+y
$$

on $[1,(g-1) / 2]$. Its maximum is at $y_{\max }=1$, with value

$$
g\left(y_{\max }\right)=(g-2)^{2}+3<(g-2)^{2}+4 .
$$


Step 3. The last case is that of products of the form $A^{k} \times B^{l}$, with $\operatorname{dim} A=a>1$, $\operatorname{dim} B=b>1, k \geq l \geq 1$ and $g=a k+b l$. One has,

$$
\begin{aligned}
\rho\left(A^{k} \times B^{l}\right) & \leq \frac{1}{2} a k(2 k+1)+\frac{1}{2} b l(2 l+1) \\
& \leq \frac{1}{2} a k(2 k+1)+\frac{1}{2} b l(2 k+1)= \\
& =\frac{1}{2} g(2 k+1) \leq \frac{1}{2} g(g-1)<(g-2)^{2}+4 .
\end{aligned}
$$

\section{Structure of abelian varieties with large Picard number}

As an application of Theorem 1.1, we will now derive a structure result for abelian varieties of large Picard number (up to isogeny). Our starting point is the following result:

Theorem 4.1 (Exercise 5.6.10 of [7]). Let $A$ be an abelian variety of dimension $g$. The following are equivalent

(1) $\rho(A)=g^{2}$;

(2) $A \sim E^{g}$, for some elliptic curve $E$ with complex multiplication;

(3) $A \cong E_{1} \times \cdots \times E_{g}$, for some pairwise isogenous elliptic curves $E_{1}, \ldots, E_{g}$ with complex multiplication.

This result points out how the Picard number can force the structure of an algebraic variety to be in some sense rigid. Algebraic varieties with the maximum Picard number possible have shown to possess interesting arithmetic and geometric properties: for example, see [20] and [19], or [5] for a recent account.

The aim of this section is to prove a similar statement for abelian varieties whose Picard number is the second or third largest attainable according to Theorem 1.1, namely $(g-1)^{2}+1$ or $(g-2)^{2}+4$. However, unlike in the case of maximal Picard number, one cannot expect a statement which is analogous to Theorem 4.1(3). Already for $\rho(A)=(g-1)^{2}+1$, one can construct abelian varieties which are isogenous to $E_{1}^{g-1} \times E_{2}$, but which are not isomorphic to a product of elliptic curves.

The following result describes the structure of these abelian varieties up to isogeny. It should be noticed that, in contrast with Theorem 4.1, the result depends on the dimension of the abelian varieties we consider: on one hand we need Theorem 1.1, and on the other we need to guarantee that the abelian varieties having such Picard numbers all belong to a unique isogeny class.

Theorem 4.2. Let $A$ be an abelian variety of dimension $g$.

(1) Suppose $g \geq 5$. Then,

$$
\rho(A)=(g-1)^{2}+1 \Longleftrightarrow A \sim E_{1}^{g-1} \times E_{2},
$$

where $E_{1}$ has complex multiplication and $E_{1}$ and $E_{2}$ are not isogeneous.

(2) Suppose $g \geq 7$. Then,

$$
\rho(A)=(g-2)^{2}+4 \Longleftrightarrow A \sim E_{1}^{g-2} \times E_{2}^{2},
$$

where $E_{1}$ and $E_{2}$ both have complex multiplication but are not isogeneous. 
Proof. Recall that we have the following (strictly) increasing sequence of positive integers:

$$
g=M_{g, g}<M_{g-1, g}<\cdots<M_{3, g}<M_{2, g}<M_{1, g}=g^{2} .
$$

Assume $\rho(A)=(g-1)^{2}+1=M_{2, g}$ and $g \geq 5$. By definition of $M_{r, g}$ it follows that $r(A) \leq 2$; we claim that $r(A)=2$. Indeed, if $r(A)=1$, then necessarily $A \sim E^{g}$. But then either $\rho(A)=g^{2}$ if $E$ has CM (by Theorem 4.1) or $\rho(A)=\left(\begin{array}{c}g+1 \\ 2\end{array}\right)$ otherwise, either of which is a contradiction. Therefore $r(A)=2$ and (1) follows from Corollary 3.2 .

Now let $A$ have Picard number $\rho(A)=(g-2)^{2}+4$, and let $g \geq 7$. As $\rho(A)>M_{3, g}$, we deduce $r(A) \leq 2$. If $r(A)=1$ we again get a contradiction as above (this time, we also use $g \geq 7$ ), hence $r(A)=2$. In a similar fashion to the proof of the Theorem 1.1, we distinguish three cases:

(a) Let $A \sim A^{k} \times B^{l}$, with $\operatorname{dim} A>1$ and $\operatorname{dim} B>1$. Then, as we have seen in Step 3 of the proof of Theorem 1.1

$$
\rho(A) \leq \frac{1}{2} g(g-1)<(g-2)^{2}+4,
$$

which gives a contradiction.

(b) Let $A \sim E^{k} \times B^{l}$, with $\operatorname{dim} B>1$ and $\operatorname{dim} E=1$. Then, as we have seen in Step 2

$$
\rho(A) \leq(g-2)^{2}+3<(g-2)^{2}+4,
$$

again a contradiction.

(c) Let $A \sim E_{1}^{n} \times E_{2}^{g-n}$, for two elliptic curves $E_{1}$ and $E_{2}$. Then, the cases $n=1$ and $n=g-1$ can be discarded, by previous discussions. Therefore, let us suppose $2 \leq n \leq g-2$. We claim that $\rho(A)<(g-2)^{2}+4$, unless both $E_{1}$ and $E_{2}$ have complex multiplication. Indeed, if one of the factors does not have complex multiplication then $\rho(A) \leq 3+(g-2)^{2}<4+(g-2)^{2}$. Therefore both $E_{1}$ and $E_{2}$ must have complex multiplication, and so $\rho(A)=\rho\left(E_{1}^{n} \times E_{2}^{g-n}\right)=n^{2}+(g-n)^{2}$. The maximum of this expression is achieved for $n=2$ or $n=g-2$, and this corresponds to a product $E_{1}^{2} \times E_{2}^{g-2}$.

We have thus shown that the only possible case is $A \sim E_{1}^{2} \times E_{2}^{g-2}$, for two non-isogeneous elliptic curves $E_{1}$ and $E_{2}$ with complex multiplication, hence proving (2), and in this case the Picard number is as stated.

Clearly, one can continue this analysis along arguments used above. However, one cannot, in general, expect to obtain a unique decomposition for a given Picard number. Already for $\rho=(g-2)^{2}+3$ there are two possible isogeny decompositions, namely:

(1) $E_{1}^{g-2} \times E_{2}^{2}, E_{1}$ being an elliptic curve with complex multiplication and $E_{2}$ not having complex multiplication;

(2) $E^{g-2} \times S, E$ being an elliptic curve with complex multiplication and $S$ being a simple abelian surface of type II (these do exist by results of Shimura [18], see also the discussion in Section [5).

Remark 4.3. The Picard numbers $g^{2}$ and $(g-2)^{2}+4$ both lead to cases which have no complex moduli, whereas the intermediate Picard number $(g-1)^{2}+1$ leads to 1-dimensional families. This is in striking contrast to the case of K3 surfaces where increasing the Picard number by one corresponds to a decrease in the number of moduli by one. This is clear from the Torelli theorem for K3 surfaces. The difference lies in the fact that the Torelli theorem 
for K3 surfaces works with a weight 2 Hodge structure, wheres abelian varieties are governed by weight 1 Hodge structures.

\section{Computing Picard numbers}

In this section we approach the question how to compute the set $R_{g}$ of possible Picard numbers of abelian varieties of a given dimension $g$. To this end, let us fix a positive integer $G$, such that we are interested in computing $R_{G}$. Because of the structure of $R_{G}$, we will in fact have to compute the sets $R_{g}$ for all $g \leq G$. In order to do this, we need to compute the Picard numbers of simple abelian varieties of dimension $g$, for every $g \leq G$.

5.1. Picard numbers of simple abelian varieties. Let $g \geq 1$ be a fixed integer. If $X$ is a simple abelian variety of dimension $g$, its Picard number $\rho=\rho(X)$ must respect some divisibilty conditions [7, Proposition 5.5.7], namely

(I) Type $I: \rho \mid g$;

(II) Type $I I: \rho \in 3 \mathbb{N}$ and $\frac{2}{3} \rho \mid g$;

(III) Type III: $2 \rho \mid g$;

$(I V)$ Type $I V: \rho \mid g$.

For a fixed dimension $g$, we would like to understand which $\rho$ satisfying condition $(I),(I I)$, ( III or $(I V)$ above can actually appear as the Picard number of a simple abelian variety of the corresponding type. For the notation used in the statement and in the proof of the next result, we refer the reader to Section 2 and to [7, Ch. 9] (in particular Section 9.6).

Proposition 5.1. Let $g$ be a fixed positive integer. For all positive integers $\rho$ that satisfy one of the conditions above, there exists a simple abelian variety $X$ of the corresponding type such that $\rho(X)=\rho$, unless we are in one of the five following exceptional cases:

(1) $F$ is of type III, and $m:=g / 2 e=1$;

(2) $F$ is of type III, $m:=g / 2 e=2$, and there exists a totally positive element $\alpha \in K$ such that $N(T)=\alpha^{2}$ ( $N$ being the reduced norm of $M_{2}(F)$ to $K$ );

(3) $F$ is of type $I V, \sum_{\nu=1}^{e_{0}} r_{\nu} s_{\nu}=0$;

(4) $F$ is of type $I V, m:=g / d^{2} e_{0}=2, d=1$ and $r_{\nu}=s_{\nu}=1$ for all $\nu=1, \ldots, e_{0}$;

(5) $F$ is of type $I V, m:=g / d^{2} e_{0}=1, d=2$ and $r_{\nu}=s_{\nu}=1$ for all $\nu=1, \ldots, e_{0}$.

Proof. It is a theorem of Shimura that given an endomorphism structure $\left(F,{ }^{\prime}, \iota\right)$ one has that a general member $(X, H, \iota)$ of the moduli space $\mathcal{A}(\mathcal{M}, T)$ has the property $\operatorname{End}_{\mathbb{Q}}(X)=\iota(F)$, except in the cases above (for example see [18], or [7] for a modern approach).

In fact, under the assumption that our abelian variety $X$ be simple, one can show that these cases never occur:

(1) $X$ is isogenous to a square $Y^{2}$, where $Y$ is an abelian variety of dimension $e_{0}$, contradicting the fact that $X$ is simple;

(2) same argument as above;

(3) $X$ is isogenous to $Y^{d^{2} m}$, where $Y$ is an abelian variety of dimension $e_{0}$, thus $d=m=1$, while $d=2$ because $F$ is a quaternion algebra over its center;

(4) $\operatorname{End}_{\mathbb{Q}}(X)$ contains a totally indefinite quaternion algebra $\tilde{F}$ over $K_{0}$ with $F=K \subset \tilde{F}$, so that $F=K \subset \tilde{F} \subset \operatorname{End}_{\mathbb{Q}}(X)=F$, contradiction;

(5) as in (1) and (2). 
For details, consult [7, Ch. 9, Ex. 9.10(1)-(5)], or see the original paper by Shimura [18]. By work of Gerritzen [9] and Albert [1]3], this implies that given an involutorial division algebra $F$ of type I-IV outside of the five exceptional cases above, there exists a simple abelian variety whose endomorphism algebra is $F$. For a survey on these results, see [14].

We are now left to show that for any integer $\rho$ satisfying one of the conditions (I-IV) and outside of (1)-(5), we can actually construct an involutorial division algebra $F$ of the corresponding type, such that there exists an abelian variety $X$ with $\operatorname{End}_{\mathbb{Q}}(X) \cong F$ and $\rho(X)=\rho$. We will divide our analysis according to the type.

5.1.1. Type $I$. Let $\rho$ be a positive integer such that $\rho \mid g$. In this case, it is enough to construct a totally real number field $F$ of degree $\rho$ over $\mathbb{Q}$. However, given a finite abelian group $G$, it is always possible to construct a totally real number field $F$ such that $\operatorname{Gal}(F / \mathbb{Q}) \cong G$ as a subfield of a suitable cyclotomic field. This implies, in particular, that we can exhibit a totally real number field of degree $\rho$ over $\mathbb{Q}$.

5.1.2. Type $I I$. It is enough to construct a totally indefinite quaternion algebra $F$ over a totally real number field $K$ of degree $e=[K: \mathbb{Q}]$ over $\mathbb{Q}$, such that $F$ is a division ring, as then $\rho=3 e$ satisfies the required condition. We are able to exhibit such an algebra for any $e \mid g$ by simply considering a quaternion algebra $F$ whose ramification is non-empty and disjoint from the archimedean place of $K$, i.e.

$$
\emptyset=\operatorname{Ram}(F) \cap\{\operatorname{archimedean} \text { places of } K\} \quad \text { and } \operatorname{Ram}(F) \neq \emptyset \text {. }
$$

5.1.3. Type III. In this situation, we aim at constructing a totally definite quaternion algebra $F$ over a totally real number field $K$ of degree $e=[K: \mathbb{Q}]$ over $\mathbb{Q}$, such that $F$ is in fact a division ring. It is enough to consider a quaternion algebra $F$ whose ramification locus is non-empty (this ensures the condition of being a division ring) and fulfills the condition

$$
\emptyset \neq \operatorname{Ram}(F) \supseteq\{\operatorname{archimedean} \text { places of } K\} \text {. }
$$

5.1.4. Type $I V$. We are left with the case corresponding to an involutorial division algebra $F$ of the second kind, whose center $K$ is a CM field with maximal real subfield $K_{0}$. In this case, it is enough to consider CM fields $K$ such that the degree $e_{0}=\left[K_{0}: \mathbb{Q}\right]$ of the maximal totally real subfield $K_{0}$ of $K$ ranges among all divisors of $g$ (i.e. we are considering the case $d=1)$.

\section{Additivity of the Range of Picard numbers}

6.1. Additivity. As before we denote by $R_{g}$ the set of realizable Picard numbers of $g$ dimensional abelian varieties, i.e.

$$
R_{g}:=\{\rho \mid \exists X \text { abelian variety, } \operatorname{dim} X=g, \rho(X)=\rho\} .
$$

Conventionally, let us set $R_{0}=\{0\}$. The main result of this section is

Proposition 6.1. For any integers $g, h \geq 0$ we have an inclusion

$$
R_{g}+R_{h}:=\left\{x+y \mid x \in R_{g}, y \in R_{h}\right\} \subset R_{g+h} .
$$


Clearly, the idea is to use the additivity of Picard numbers for products of non-isogeneous abelian varieties, as proven in Corollary 2.3. In order to be able to us this we need that for any $k \in R_{g}$ we find countably many abelian varieties of dimension $g$ and Picard number $k$ in different isogeny classes. The case of elliptic curves illustrates how this can be proved: Suppose $E$ and $E^{\prime}$ are two elliptic curves, and let $F:=\operatorname{End}_{\mathbb{Q}}(E)$ and $\operatorname{End}_{\mathbb{Q}}\left(E^{\prime}\right)$ be their endormorphism algebras. If $E$ and $E^{\prime}$ have CM, then they are mutually isogenous if and only if $F \cong F^{\prime}$. However, if they don't have $\mathrm{CM}$, then $F \cong F^{\prime} \cong \mathbb{Q}$ but $E$ and $E^{\prime}$ are not necessarily isogenous. However, by removing $\mathrm{CM}$ elliptic curves from $\mathcal{M}_{1,1}$, we get an uncountable set of isomorphism classes of elliptic curves. Since each isogeny class consists of countably many (isomorphism classes of) elliptic curves, we must have infinitely many isogeny classes of non-CM elliptic curves. We first note

Proposition 6.2. Let $X$ and $X^{\prime}$ be two simple abelian varieties of dimension $g$, and let $F$ and $F^{\prime}$ be the corresponding endomorphisms algebras. Then, if $X$ is isogenous to $X^{\prime}$, then $F \cong F^{\prime}$.

Proof. The isomorphism $\iota: \operatorname{End}_{\mathbb{Q}} X^{\prime} \longrightarrow \operatorname{End}_{\mathbb{Q}} X$ is defined by sending $\alpha \longmapsto \psi \circ \alpha \circ \phi$, where $\phi: X \longrightarrow X^{\prime}$ is an isogeny, and $\psi: X^{\prime} \longrightarrow X$ is the unique isogeny such that $\psi \circ \phi=e_{X}$ and $\phi \circ \psi=e_{Y}, e$ being the exponent of $\phi$ (or $\psi$ respectively). Surjectivity and injectivity of $\iota$ follow from the fact that multiplication maps are invertible in the endomorphism $\mathbb{Q}$ algebra.

The key result of this section is the

Proposition 6.3. Given an integer $k \in R_{g}$, then there exist at least countably many isogeny classes of abelian varietiss of dimension $g$ and Picard number $k$.

Proof. Assume that $k \in R_{g}$ and that this integer is realized by an abelian variety $A$ of dimension $g$. We first assume that $F:=\operatorname{End}_{\mathbb{Q}}(X) \neq \mathbb{Q}$. We will now go through the various types of endomorphism algebras and start with type $I$ and $[F: \mathbb{Q}]>1$.

For type I, the endomorphism algebra is a totally real number field $F$, and the Picard number of a simple abelian variety with such an endomorphism algebra is the degree $e:=$ $[F: \mathbb{Q}]$ of $F$. One can find infinitely many totally real number fields of a fixed degree $e>1$. By taking the corresponding (infinitely many) abelian varieties, we are done.

For type II-III, the endomorphism algebra is a quaternion algebra $F$ over a totally real number field $K$. In this case, the Picard number is $\rho=3 e$ for type II and $\rho=e$ for type III, where $e:=[K: \mathbb{Q}]$. Since by the previous argument there exist infinitely many totally real number fields of degree $e$, it follows that we can find infinitely many (totally definite or totally indefinite) quaternion algebras. By considering the corresponding abelian varieties, we have shown the claim for types II-III.

For type IV, the endomorphism algebra $F$ has degree $[F: K]=d^{2}$ over a CM field $K$, $e:=[K: \mathbb{Q}]$. If $K_{0}$ is the totally real subfield of $K$, of degree $e_{0}:=\left[K_{0}: \mathbb{Q}\right]$, the Picard number is $\rho=e_{0} d^{2}$. Similarly to the argument in the proof of Proposition 5.1, we can restrict ourselves to consider abelian varieties whose endomorphism algebra satisfies the condition $d=1$ : under this assumption, $\rho=e_{0}$. Since there are infinitely many totally real number fields, we find infinitely many CM fields (by just adding the imaginary unit), and the same argument as in the previous cases shows the statement for type IV abelian varieties.

This leaves us with the general situation where $F=\operatorname{End}_{\mathbb{Q}}(X) \cong \mathbb{Q}$. The ppav of this type are given by removing from $\mathcal{A}_{g}$ a countable union of proper Shimura varieties. Since 
$\mathcal{A}_{g}$ has positive dimension and since the set of ppav isogeneous to a given abelian variety is countable, the claim follows.

Proof of Proposition 6.1. This now follows immediately from Proposition 6.3 and the additivity proved in Corollary 2.3 .

6.2. Computing $R_{g}$. Our final aim is to find all realizable Picard number of abelian varieties of a given dimension. We can use Proposition 6.1 to easily show that some of the lower one indeed occur.

Proposition 6.4. Given $g \geq 2$, consider the set $R_{g}$ of Picard numbers of abelian varieties of dimension $g$. Then, $\{1, \ldots, 2 g\} \subset R_{g}$.

Proof. As $R_{2}=\{1, \ldots, 4\}$ is complete and $R_{3} \supset\{1, \ldots, 6\}$, the result easily follows by induction on $g$.

Remark 6.5. In fact, it is not hard to prove that all Picard numbers $\rho$ satisfying the inequality $g \leq \rho \leq 2 g$ are attained by products of elliptic curves.

This now allows us to formulate an algorithm which computes the ranges $R_{g}$ inductively.

- set $R_{1}=\{1\}$ and $R_{2}=\{1,2,3,4\}$;

- for all $g$ in the range $3 \leq g \leq G$, we compute $R_{g}$ as follows:

(i) by Proposition 6.4, $R_{g} \supset\{1, \ldots, 2 g-1\}$ (in particular, all Picard numbers of simple abelian varieties of dimension $g$ are in this range);

(ii) compute all possible Picard numbers of self-product abelian varieties $A^{k}$, where $\operatorname{dim} A=g / k$

(iiii) for every pair $\left(g_{1}, g_{2}\right)$ of positive integers such that $g_{1}+g_{2}=g$, compute $R_{g_{1}}+R_{g_{2}}$;

(iv) assemble everything in light of

$$
R_{g}=\bigcup_{k \mid g}\left\{\rho\left(A^{k}\right) \mid A \text { simple, } \operatorname{dim} A=g / k\right\} \cup \bigcup_{1 \leq n \leq g-1}\left(R_{n}+R_{g-n}\right) .
$$

\section{Asymptotic Behaviour of Picard numbers of ABelian VARieties}

7.1. Asymptotic completeness of Picard numbers. In the course of this note, we have shown that for every $g \geq 3$ the set $R_{g}$ of Picard numbers of $g$-dimensional abelian varieties is not complete, or in other words that $\# R_{g}<g^{2}$. The ratio $\delta_{g}:=\# R_{g} / g^{2}$ is the density of $R_{g}$ in $\left[1, g^{2}\right] \cap \mathbb{N}$, and it describes how many admissible Picard numbers (according to Lefschetz Theorem of $(1,1)$-classes) can actually be attained. One may wonder about the asymptotic density of Picard numbers of abelian varieties: this is the quantity defined as

$$
\delta:=\lim _{g \rightarrow+\infty} \delta_{g}
$$

We now show that the Picard numbers of abelian varieties are asymptotically complete, namely that $\delta=1$, contrary to the fact that $\delta_{g}<1$ for every $g \geq 3$.

Theorem 7.1 (Asymptotic completeness). The sets of Picard numbers of abelian varieties are asymptotically dense, i.e. $\delta=1$.

The proof relies on Lagrange's four-square theorem and the following lemma, whose proof follows readily from the additivity of the Picard number. 
Lemma 7.2. Suppose $g \geq 1$ and $1 \leq n \leq g^{2}$, where $g$ and $n$ are two integers. Assume that there exist positive integers $n_{1}, \ldots, n_{k}$ such that

$$
n-1=n_{1}^{2}+\cdots+n_{k}^{2} \quad \text { and } \quad n_{1}+\cdots+n_{k} \leq g-1 .
$$

Then, there exists a $g$-dimensional abelian variety $X$ with $\rho(X)=n$.

Proof of Proposition 7.1. Let $n_{1}$ be the largest positive integer such that

$$
n_{1}^{2} \leq n-1<\left(n_{1}+1\right)^{2} .
$$

Then,

$$
0 \leq n-1-n_{1}^{2}<\left(n_{1}+1\right)^{2}-n_{1}^{2}=2 n_{1}+1,
$$

from which it follows that

$$
0 \leq n-1-n_{1}^{2} \leq 2 n_{1} \leq 2 \sqrt{n-1}<2 \sqrt{n} \leq 2 g .
$$

Lagrange's four-square theorem implies that

$$
m:=n-1-n_{1}^{2}=n_{2}^{2}+n_{3}^{2}+n_{4}^{2}+n_{5}^{2},
$$

for some $n_{2}, n_{3}, n_{4}, n_{5} \in \mathbb{N}$. We will now show that $n_{1}+\cdots+n_{5}<g$ for $g \gg 0$ : indeed, by looking at the power means of $n_{2}, \ldots, n_{5}$ one has that

$$
\frac{n_{2}+\cdots+n_{5}}{4} \leq \sqrt{\frac{n_{2}^{2}+n_{3}^{2}+n_{4}^{2}+n_{5}^{2}}{4}}=\frac{1}{2} \sqrt{m} \leq \frac{1}{2} \sqrt{2 g} .
$$

Therefore,

$$
n_{1}+\cdots+n_{5} \leq \sqrt{n-1}+2 \sqrt{2 g},
$$

and the right-hand side is strictly smaller than $g$ if and only if

$$
n<g^{2}+8 g+1-4 \sqrt{2} g^{3 / 2}=: b_{g} .
$$

This implies that all Picard numbers in the range $\left[1, b_{g}\right)$ indeed occur, by virtue of the lemma above. Hence, we have that asymptotically $\# R_{g} \geq b_{g}-1=g^{2}+8 g-4 \sqrt{2} g^{3 / 2}$, and thus $\delta=1$.

7.2. Distribution of large Picard numbers. We are interested in describing the distribution of large Picard numbers within $\left[1, g^{2}\right] \cap \mathbb{N}$. As we have already observed, for every $g \geq 1$, the set $R_{g}$ has the following structure

$$
R_{g}=\bigcup_{k \mid g}\left\{\rho\left(A^{k}\right) \mid A \text { simple, } \operatorname{dim} A=g / k\right\} \cup \bigcup_{1 \leq n \leq g-1}\left(R_{n}+R_{g-n}\right) .
$$

The proof of Theorem 1.1(1) shows that all Picard numbers of abelian varieties of dimension $g$ that are isogenous to a self-product of a simple abelian variety are bounded by $\frac{1}{2} g(g+1)$, unless we are considering the $g$-fold product of a CM elliptic curve, in which case the maximal Picard number is attained.

In order to begin our analysis, we need to specify what we mean by "large Picard numbers". First of all, we will require large Picard numbers to satisfy the inequality $\rho>g(g+1) / 2$. In particular, this implies that we need not concern ourselves with those abelian varieties whose isogeny decomposition only has one factor. Therefore, we can focus on the following subset of $R_{g}$ :

$$
\bigcup_{1 \leq n \leq g-1}\left(R_{n}+R_{g-n}\right)
$$


Now we need a little bit of notation. Let us set

$$
R_{g, n}:=\left\{(g-n)^{2}+x \mid x \in R_{n}\right\} .
$$

In other words, $R_{g, n}$ is the subset of $R_{g}$ obtained by translating $R_{n}$ to the right by $(g-n)^{2}$ inside $\mathbb{N}$. Notice that given $g, k, n \in \mathbb{N}$, one has by Proposition [6.1 that

$$
R_{g, k}+R_{n} \subset R_{g+n, k+n} .
$$

As we want to consider large Picard numbers only, we will be concerned only with some of the $R_{g, s}$ 's, namely those for which the inequality

$$
\frac{1}{2} g(g+1) \leq(g-s)^{2}+1
$$

holds $(1 \leq s \leq g)$, which implies that the abelian varieties we are considering are not selfproducts of simple abelian varieties. This in particular implies that $g \geq 4$ (because $s \geq 1$ ) and

$$
s \leq \frac{2 g-\sqrt{2\left(g^{2}+g-2\right)}}{2} .
$$

Finally, let us look at the mutual interaction of the $R_{g, s}$ 's. For a fixed $g$, there might exist positive integers $a$ and $b$ such that $R_{g, a} \cap R_{g, b} \neq \emptyset$. However, if $a$ and $b$ are distinct and small enough with respect to $g$, then $R_{g, a} \cap R_{g, b}=\emptyset$. Indeed, for a fixed $g$, the inequality

$$
[g-(s+1)]^{2}+(s+1)^{2}<(g-s)^{2}+1
$$

holds for all positive integers $s<-2+\sqrt{2 g+3}$. Hence $R_{g, a} \cap R_{g, b}=\emptyset$ for $a$ and $b$ in this range.

We are now able to define precisely what "large Picard number" means. We will say that a Picard number $\rho$ is large if $\rho \in R_{g, s}$ with $s$ satisfying conditions (1) and (2). This condition can be made explicit:

Proposition 7.3. Let $g \geq 4$. Then, $\rho \in R_{g, s}$ is a large Picard number if and only if

$$
s \leq \min \left\{\frac{2 g-\sqrt{2\left(g^{2}+g-2\right)}}{2},-1+\sqrt{2 g+3}\right\} .
$$

The theorem we are going to discuss next describes the distribution of the large Picard numbers inside $\left[1, g^{2}\right] \cap \mathbb{N}$. The argument we use is inductive and its initial step is a proof of an asymptotic version of Theorem 1.1(2) starting from an asymptotic version of Theorem 1.1(1).

Theorem 7.4 (Distribution of large Picard numbers). For every positive integer $\ell$ there exists a genus $g_{\ell}$ such that for all $g \geq g_{\ell}$ large Picard numbers in $R_{g}$ are distributed as follows:

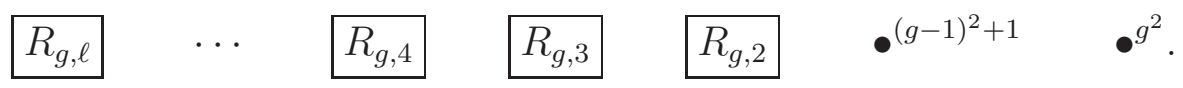

In other words, for all $g \geq g_{\ell}$, we have that

$$
\left[(g-\ell)^{2}+1, g^{2}\right] \cap R_{g}=R_{g, \ell} \sqcup R_{g, \ell-1} \sqcup \cdots \sqcup R_{g, 2} \sqcup R_{g, 1} \sqcup R_{g, 0} .
$$

Remark 7.5. In particular this shows that, as $g \rightarrow \infty$ more and more gaps arise in $R_{g}$ as we go down from the maximum Picard number $g^{2}$. 
Proof. We will give a proof by induction. To start with, note that we can (and will) always assume that $g$ is large enough, so that there is no overlapping between the sets $R_{g, n}$ that we wish to consider.

Let us start with a pair $\left(\ell, g_{\ell}\right)$ such that the following holds: there is no abelian variety of dimension $g \geq g_{\ell}$ and Picard number $\rho$ such that $(g-t)^{2}+t^{2}<\rho<(g-t+1)^{2}+1$ for $2 \leq t \leq \ell$ or $(g-1)^{2}+1<\rho<g^{2}$. Then, the claim is that we can find $g_{\ell+1} \geq g_{\ell}$ such that there is no abelian variety of dimension $g \geq g_{\ell+1}$ and Picard number $\rho$ such that $[g-(\ell+1)]^{2}+(\ell+1)^{2}<\rho<(g-\ell)^{2}+1$.

As the start of the induction, we will now show how to recover the second part of Theorem 1.1 from the first one, at least asymptotically (we will not be able to get any bound on $g$, but of course it is always possible to do so). Suppose that for all $g \geq g_{1}$ there is no abelian variety $X$ of dimension $g$ and Picard number $(g-1)^{2}+1<\rho(X)<g^{2}$ (notice that in light of Theorem 1.1. we can choose $\left.g_{1}=4\right)$. We will now prove that there exists $g_{2}, g_{2} \geq g_{1}$, such that for every $g \geq g_{2}$ there is no abelian variety $Y$ of dimension $g$ and Picard number $(g-2)^{2}+4<\rho(Y)<(g-1)^{2}+1$.

Let $g_{2}$ be such that for all $g \geq g_{2}$ conditions (1) and (2) above are satisfied (i.e. the Picard numbers $\rho$ in the range $(g-2)^{2}+4<\rho<(g-1)^{2}+1$ are large according to our definition). Suppose $Y$ is an abelian variety whose Picard number contradicts the statement we want to prove, namely $(g-2)^{2}+4<\rho(Y)<(g-1)^{2}+1$. Then, as $\rho(Y)$ is large, $Y$ is isogenous to a product of two abelian varieties, i.e. $Y \sim A_{n} \times A_{g-n}$, where $n \leq g-n$ and $\operatorname{Hom}\left(A_{n}, A_{g-n}\right)=0$, (here the subscripts indicate the dimension). Since $\rho>(g-2)^{2}+4$, we have that $n=1$ necessarily. Therefore $Y \sim E \times A_{g-1}$, where $E$ is an elliptic curve and $\operatorname{Hom}\left(E, A_{g-1}\right)=0$. As $\rho(Y)=1+\rho\left(A_{g-1}\right)$, we readily see that

$$
(g-2)^{2}+1<\rho\left(A_{g-1}\right)<(g-1)^{2},
$$

a contradiction. This is first step of the induction.

Now, let us assume that there exists $g_{\ell}$ such that for all $g \geq g_{\ell}$ there is no abelian variety $X$ of dimension $g$ and Picard number in the following ranges:

$$
\begin{aligned}
& \text { (1) }(g-1)^{2}+1<\rho(X)<g^{2} \text {; } \\
& \text { (2) }(g-2)^{2}+4<\rho(X)<(g-1)^{2}+1 \text {; } \\
& \text { ( } \ell)(g-\ell)^{2}+\ell^{2}<\rho(X)<[g-(\ell-1)]^{2}+1 \text {. }
\end{aligned}
$$

We claim that there exists $g_{\ell+1}$ such that for all $g \geq g_{\ell+1}$ there is no abelian variety $Y$ of dimension $g$ and Picard number satisfying

$$
(\ell+1)[g-(\ell+1)]^{2}+(\ell+1)^{2}<\rho(Y)<[g-\ell]^{2}+1 .
$$

Again, let us let $g$ grow bigger so that the Picard numbers we wish to consider can only be realized by abelian varieties that are not a self-product of a simple abelian variety. By contradition, let $Y$ be an abelian variety that contradicts the statement we want to prove. Then $Y \sim A_{n} \times A_{g-n}$, where $n \leq g-n$ and $\operatorname{Hom}\left(A_{n}, A_{g-n}\right)=0$. It is straightforward to see that $n \leq \ell$, as $\rho(Y)>[g-(\ell+1)]^{2}+(\ell+1)^{2}$. By additivity of the Picard number

$$
\rho(Y)=\rho\left(A_{n} \times A_{g-n}\right)=\underbrace{\rho\left(A_{n}\right)}_{\rho_{n}}+\underbrace{\rho\left(A_{g-n}\right)}_{\rho_{g-n}} .
$$


As $\rho(Y)>[g-(\ell+1)]^{2}+(\ell+1)^{2}$, we see that

$$
\begin{aligned}
\rho_{g-n} & >[g-(\ell+1)]^{2}+(\ell+1)^{2}-\rho_{n}>[g-(\ell+1)]^{2}+(\ell+1)^{2}-n^{2} \\
& >[(g-n)-(\ell-n+1)]^{2}+(\ell+1)^{2}-n^{2} \\
& >[(g-n)-(\ell-n+1)]^{2}+(\ell-n+1)^{2} .
\end{aligned}
$$

Similarly,

$$
\rho_{g-n}<(g-\ell)^{2}+1-\rho_{n} \leq(g-\ell)^{2}=[(g-n)-(\ell-n)]^{2}<[(g-n)-(\ell-n)]^{2}+1,
$$

and summing up we have shown that

$$
[(g-n)-(\ell-n+1)]^{2}+(\ell-n+1)^{2}<\rho\left(A_{g-n}\right)<[(g-n)-(\ell-n)]^{2}+1,
$$

which contradicts the $(\ell-n+1)$-st condition above.

As a striking consequence of Theorem 7.4, we get the following structure theorem for abelian varieties of large Picard number up to isogeny, which generalizes the results in Section 4. As we had already noticed in Section 4. we cannot expect a structure theorem up to isomorphism, hence this is the strongest result we could hope for.

Corollary 7.6 (Structure theorem for abelian varieties of large Picard number). For every positive integer $\ell$ there exists a genus $g_{\ell}$ such that for all $g \geq g_{\ell}$ the following are equivalent:

(1) $\rho(X) \in R_{g, n}$ for some $n \leq \ell$;

(2) $X \sim E_{g-n} \times A_{n}$, where $E$ is an elliptic curve with complex multiplication, $A_{n}$ is an abelian variety of dimension $n$, and $\operatorname{Hom}\left(E, A_{n}\right)=0$.

Proof. Let us set $g_{\ell}$ as in the proof of Theorem [7.4, and let $X$ be an abelian variety of Picard number $\rho(X) \in R_{g, n}$ for some $n \leq \ell$. By means of the Poincaré reducibility theorem, we can write $X \sim E^{t} \times A_{g-t}$, where $E$ is an elliptic curve with complex multiplication, $A_{g-t}$ is an abelian variety of dimension $g-t, \operatorname{Hom}\left(E, A_{g-t}\right)=0$, and $t$ is the largest integer appearing as exponent of an elliptic curve with complex multiplication in the isogeny decomposition of $X$. Let us now set for simplicity $t=g-m$, so that $X \sim E^{g-m} \times A_{m}$. In particular, it follows that $\rho(X) \in R_{g, m}$. However, by Theorem 4.2, $R_{g, n}$ cannot intersect $R_{g, m}$ unless $n=m$, from which the statement follows.

\section{ABELIAN VARIETIES DEFINED OVER NUMBER FIELDS}

In this section we show that every realizable Picard number $\rho \in R_{g}$ can be obtained by an abelian variety defined over a number field.

Theorem 8.1. Let $(X, \lambda)$ be a polarized complex abelian variety, let $D=\operatorname{End}^{0}(X)$ be the endomorphism algebra of $X$ and let $*$ be the Rosati involution on $D$. Then there exists a polarized abelian variety over $\overline{\mathbb{Q}}$, or equivalently over a number field, which has the same endomorphism algebra with involution $(D, *)$.

Proof (Ben Moonen). To prove the assertion, choose a $\mathbb{Q}$-subalgebra $R \subset \mathbb{C}$ of finite type and a polarized abelian scheme $(Y, \mu)$ over $S:=\operatorname{Spec}(R)$ such that $(Y, \mu) \otimes_{R} \mathbb{C}$ is isomorphic to $(X, \lambda)$ and such that all endomorphisms of $X$ are defined over $R$, in the sense that the natural map

$$
\operatorname{End}^{0}(Y / R) \longrightarrow \operatorname{End}^{0}(X)
$$


is an isomorphism. The existence of such a model follows from [10, Proposition (8.9.1)] together with the fact that $\operatorname{End}(X)$ is a finitely generated algebra (in fact, it is even finitely generated as an abelian group). By construction, if $\eta$ is the generic point of $S$, we have $\operatorname{End}^{0}\left(Y_{\eta}\right) \cong D$ as algebras with involution. If $s$ is a point of $S$, we have a specialization homomorphism $i_{s}: \operatorname{End}^{0}\left(Y_{\eta}\right) \hookrightarrow \operatorname{End}^{0}\left(Y_{s}\right)$, and we are done if we can find a closed point $s$ for which $i_{s}$ is an isomorphism.

Let $\ell$ be a prime number. For $s$ a point of $S$, let $T_{\ell}(s):=T_{\ell}\left(Y_{s}\right)$ denote the $\ell$-adic Tate module of $Y_{s}$, and let

$$
\rho_{s}: \operatorname{Gal}(\overline{\kappa(s)} / \kappa(s)) \rightarrow \operatorname{GL}\left(V_{\ell}(s)\right)
$$

denote the Galois representation on $V_{\ell}(s)=T_{\ell}(s) \otimes_{\mathbb{Z}} \mathbb{Q}_{\ell}$. By a result of Faltings [8, Theorem 1], $\operatorname{End}^{0}\left(Y_{s}\right) \otimes \mathbb{Q}_{\ell}$ is the endomorphism algebra of $V_{\ell}(s)$ as a Galois representation, namely

$$
\operatorname{End}^{0}\left(Y_{s}\right) \otimes \mathbb{Q}_{\ell} \cong \operatorname{End}_{\operatorname{Gal}(\overline{\kappa(s)} / \kappa(s))}\left(V_{\ell}(s)\right) \text {. }
$$

For $s \in S$, the image of $\rho_{s}$ may be identified with a subgroup of $\operatorname{im}\left(\rho_{\eta}\right)$; the subgroup we obtain is independent of choices only up to conjugacy. By a result of Serre [17] (see also [13, Proposition 1.3]), there exist closed points $s \in S$ for which $\operatorname{im}\left(\rho_{s}\right)=\operatorname{im}\left(\rho_{\eta}\right)$, and for all such points the specialization map $i_{s}$ on endomorphism algebras is an isomorphism (see [13, Corollary 1.5]).

As the Picard number only depends on $(D, *)$ this immediately implies

Corollary 8.2. Every realizabe Picard number $\rho \in R_{g}$ can be obtained by an abelian variety defined over a number field.

\section{REFERENCES}

[1] A. Adrian Albert. On the construction of Riemann matrices. I. Ann. of Math. (2), 35(1):1-28, 1934.

[2] A. Adrian Albert. A solution of the principal problem in the theory of Riemann matrices. Ann. of Math. (2), 35(3):500-515, 1934.

[3] A. Adrian Albert. On the construction of Riemann matrices. II. Ann. of Math. (2), 36(2):376-394, 1935.

[4] A. Beauville. Variétés Kähleriennes dont la première classe de Chern est nulle. J. Differential Geom., 18(4):755-782 (1984), 1983.

[5] Arnaud Beauville. Some surfaces with maximal Picard number. J. Éc. polytech. Math., 1:101-116, 2014.

[6] C. Birkenhake and H. Lange. Complex tori, volume 177 of Progress in Mathematics. Birkhäuser Boston, Inc., Boston, MA, 1999.

[7] C. Birkenhake and H. Lange. Complex abelian varieties, volume 302 of Grundlehren der Mathematischen Wissenschaften [Fundamental Principles of Mathematical Sciences]. Springer-Verlag, Berlin, second edition, 2004.

[8] Gerd Faltings. Complements to Mordell. In Rational points (Bonn, 1983/1984), Aspects Math., E6, pages 203-227. Vieweg, Braunschweig, 1984.

[9] L. Gerritzen. On multiplication algebras of Riemann matrices. Math Ann, 194:109-122, 1971.

[10] A. Grothendieck. Éléments de géométrie algébrique. IV: Étude locale des schémas et des morphismes de schémas. III. Inst. Hautes Études Sci. Publ. Math., (28):255, 1966.

[11] D. Huybrechts. Compact hyper-Kähler manifolds: basic results. Invent. Math., 135(1):63-113, 1999.

[12] V. Kumar Murty. Exceptional Hodge classes on certain abelian varieties. Math. Ann., 268(2):197-206, 1984.

[13] Rutger Noot. Abelian varieties - Galois representation and properties of ordinary reduction. Compositio Math., 97(1-2):161-171, 1995. Special issue in honour of Frans Oort.

[14] Frans Oort. Endomorphism algebras of abelian varieties. In Algebraic geometry and commutative algebra, Vol. II, pages 469-502. Kinokuniya, Tokyo, 1988. 
[15] M. Schütt. Quintic surfaces with maximum and other Picard numbers. J. Math. Soc. Japan, 63(4):11871201, 2011.

[16] M. Schütt. Picard numbers of quintic surfaces. Proc. Lond. Math. Soc. (3), 110(2):428-476, 2015.

[17] J.-P. Serre. Lettre á Ken Ribet du 1/1/1981. In Euvres, volume IV.

[18] Goro Shimura. On analytic families of polarized abelian varieties and automorphic functions. Ann. of Math. (2), 78:149-192, 1963.

[19] T. Shioda and H. Inose. On singular K3 surfaces. In Complex analysis and algebraic geometry, pages 119-136. Iwanami Shoten, Tokyo, 1977.

[20] T. Shioda and N. Mitani. Singular abelian surfaces and binary quadratic forms. In Classification of algebraic varieties and compact complex manifolds, pages 259-287. Lecture Notes in Math., Vol. 412. Springer, Berlin, 1974.

[21] Tetsuji Shioda. Algebraic cycles on certain K3 surfaces in characteristic $p$. pages 357-364, 1975.

KLAus HuleK

Institut für Algebraische Geometrie, Leibniz Universität Hannover, Welfengarten 130167 HANNOVER (GERMANY)

E-mail address: hulek@math.uni-hannover.de

Roberto LAFACE

Technische Universität München, Zentrum Mathematik - M11, Boltzmannstrasse 3, D-85478 Garching Bei München (Germany)

E-mail address: laface@ma.tum.de 